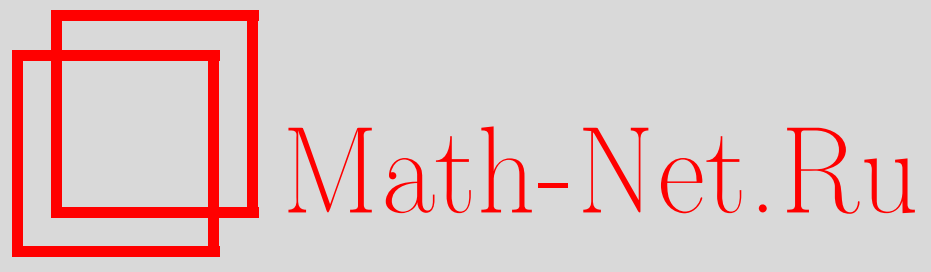

К. Б. Алкалаев, Безмассовые калибровочные поля смешанного типа симметрии в $A d S_{5}, T M \Phi, 2006$, том 149 , номер 1, 47-59

DOI: https://doi.org/10.4213/tmf3829

Использование Общероссийского математического портала Math-Net.Ru подразумевает, что вы прочитали и согласны с пользовательским соглашением http://www . mathnet.ru/rus/agreement

Параметры загрузки:

IP : 54.237 .59 .107

26 апреля 2023 г., 16:39:31

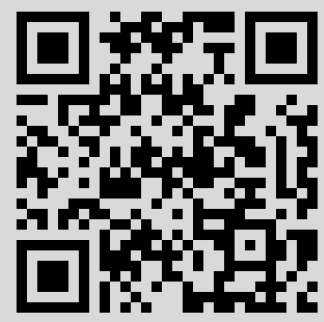




\title{
БЕЗМАССОВЫЕ КАЛИБРОВОЧНЫЕ ПОЛЯ СМЕШАННОГО ТИПА СИММЕТРИИ В $A d S_{5}$
}

\begin{abstract}
С использованием $s u(2,2)$-спинорного языка описаны свободные безмассовые бозонные и фермионные калибровочные поля смешанного типа симметрии с произвольными спинами на пятимерном пространстве анти-де Ситтера. Построены явно ковариантные функционалы действия и выведены полевые уравнения.
\end{abstract}

Ключевые слова: высшие спины, смешанный тип симметрии, пространства анти-де Ситтера, диаграммы Юнга.

\section{1. ВВЕДЕНИЕ}

В настоящей работе мы продолжаем изучение пятимерной калибровочной теории полей высших спинов [1]-[5]. Нашей целью является формулировка явно ковариантных калибровочно-инвариантных действий, описывающих свободную полевую динамику безмассовых бозонных и фермионных калибровочных полей смешанного типа симметрии с произвольными спинами $\mathbf{s}=\left(s_{1}, s_{2}\right)$.

Наше рассмотрение калибровочных полей смешанного типа симметрии на пятимерном пространстве анти-де Ситтера $\left(A d S_{5}\right)$ основано на реперном подходе, предложенном в работах [6], [7]. Однако вместо (спин-)тензорного языка, использованного в [6], [7], мы пользуемся спинорным описанием $A d S_{5}$-полей высших спинов, основанным на хорошо известном факте: $A d S_{5}$-алгебра $о(4,2)$ изоморфна алгебpe $s u(2,2)$. Тем самым $o(4,2)$-(спин-)тензорные поля могут быть эквивалентным образом описаны как $s u(2,2)$-мультиспиноры, и главное преимущество спинорного описания состоит в том, что бозонные и фермионные поля произвольного спина $\mathbf{s}=\left(s_{1}, s_{2}\right)$ могут рассматриваться единым образом. Несмотря на тот факт, что $d$-мерный анализ работ [6], [7], конечно же, включает случай $A d S_{5}$, переформулировка этих результатов на спинорном языке может быть интересна в нескольких отношениях. В целом она мотивирована желанием продвинуться в направлении нелинейной калибровочной теории полей высших спинов, которая вызывает интерес в контексте версии $A d S_{5} / C F T_{4}$-соответствия с учетом полей высших спинов (см. обзоры [8]). На самом деле, на данный момент построены нелинейные уравнения

\footnotetext{
*Физический институт им. П. Н. Лебедева РАН, Москва, Россия. E-mail: alkalaev@lpi.ru
} 
движения для $A d S_{d}$ полностью симметричных бозонных полей и отвечающие им калибровочные алгебры высших спинов [9], однако проблема их расширения на случай полей смешанного типа симметрии общего вида остается открытой. С другой стороны, в случае $A d S_{5}$-динамики высших спинов существует реальная возможность построить нелинейную теорию полей высших спинов всех типов симметрии. Действительно, в пяти измерениях имеются преимущества использования изоморфизма $o(4,2) \sim s u(2,2)$. В частности, в рамках спинорного языка (суперсимметричные) алгебры высших спинов были отождествлены с определенными *-алгебрами с $s u(2,2)$-спинорными образующими элементами [10], [11]. Также существуют явно ковариантные формулировки свободной $A d S_{5}$-динамики высших спинов ${ }^{1)}$ [1]-[4] и $\mathcal{N}=0,1$ (суперсимметричные) функционалы действия, которые описывают кубические взаимодействия $A d S_{5}$ полностью симметричных полей [2], [5].

Статья имеет следующую структуру. В разделе 2 описана фоновая $A d S_{5}$-геометрия в спинорных обозначениях [2]. В разделе 3 рассмотрены $A d S_{5}$ калибровочные поля высших спинов смешанного типа симметрии и описаны их мультиспинорная и (спин-)тензорная формы. В разделе 4 вводятся тензоры высших рангов как функции вспомогательных спинорных переменных [2], строятся калибровочные преобразования и линеаризованные кривизны высших спинов. В разделе 5 построены явно калибровочно инвариантные действия высших спинов. В разделе 6 выводятся уравнения движения и обсуждаются связи для экстра полей. Заключительные замечания приведены в разделе 7.

\section{2. $\mathrm{AdS}_{5}$ ФОНОВАЯ ГЕОМЕТРИЯ В СПИНОРНЫХ ОБОЗНАЧЕНИЯХ}

Гравитационные поля ${ }^{2)}$ в $A d S_{5}$ отождествляются с 1-формой связности, которая принимает значения в $A d S_{5}$-алгебре $s u(2,2)$

$$
\Omega(x)=d x^{\underline{n}} \Omega_{\underline{n}}{ }_{\beta} t_{\alpha}{ }^{\beta},
$$

где $t_{\alpha}{ }^{\beta}$ являются базисными элементами $s u(2,2)$ и $\Omega^{\alpha}{ }_{\alpha}=0 . s u(2,2)$-Калибровочное поле (2.1) разлагается на поле репера и лоренцеву спиновую связность. Это разбиение может быть выполнено явно $s u(2,2)$-ковариантным способом посредством введения поля компенсатора [2], [15], который является антисимметричным биспинором $V^{\alpha \beta}=-V^{\beta \alpha}$. Компенсатор нормирован так, что $V_{\alpha \gamma} V^{\beta \gamma}=\delta_{\alpha}^{\beta}, V_{\alpha \beta}=(1 / 2) \varepsilon_{\alpha \beta \gamma \rho} \times$ $V^{\gamma \rho}$. Подалгебра Лоренца отождествляется с алгеброй стабильности компенсатора.

\footnotetext{
1) Уравнения движения для $A d S_{d}$ безмассовых полей произвольных спинов в калибровке светового конуса были построены в работах [12]; действия в калибровке светового конуса для произвольных $A d S_{5}$ безмассовых полей смешанного типа симметрии - в работе [13]. Также существуют различные явно ковариантные лагранжевы формулировки для частных примеров $A d S_{d^{-}}$ калибровочных полей смешанного типа симметрии [14].

${ }^{2)} \mathrm{B}$ данной работе мы пользуемся сигнатурой $(+-\cdots-)$ и обозначениями $\alpha, \beta, \gamma=1,2,3,4$ для $s u(2,2)$-спинорных индексов, $\underline{m}, \underline{n}=0,1,2,3,4$ для мировых индексов, $a, b, c=0,1,2,3,4$ для касательных лоренцевых $s o(4,1)$-векторных индексов и $A, B, C=0,1,2,3,4,5$ для касательных $s o(4,2)$-векторных индексов. Мы также используем конденсированные обозначения для набора симметричных спинорных индексов $\alpha(k) \equiv \alpha_{1} \ldots \alpha_{k}$. Индексы, обозначенные одинаковой буквой, считаются симметризованными как $X^{\alpha} Y^{\alpha} \equiv X^{\alpha_{1}} Y^{\alpha_{2}}+X^{\alpha_{2}} Y^{\alpha_{1}}$.
} 
Это позволяет определить поле репера $E^{\alpha \beta}$ и лоренцеву спиновую связность $\omega^{\alpha(2)}$ следующим образом [2]:

$$
\begin{gathered}
E^{\alpha \beta}=D V^{\alpha \beta} \equiv d V^{\alpha \beta}+\Omega^{\alpha}{ }_{\gamma} V^{\gamma \beta}+\Omega^{\beta}{ }_{\gamma} V^{\alpha \gamma}, \quad E^{\alpha \beta} V_{\alpha \beta}=0, \\
\omega^{\alpha}{ }_{\beta}=\Omega^{\alpha}{ }_{\beta}+\frac{\lambda}{2} E^{\alpha \gamma} V_{\gamma \beta},
\end{gathered}
$$

где $\lambda$ - космологический параметр, $\lambda^{2}>0$. Отметим, что поскольку компенсатор является лоренц-инвариантным, мы рассматриваем $V^{\alpha \beta}$ как симплектическую метрику, позволяющую поднимать и опускать спинорные индексы лоренц-ковариантным образом: $X^{\alpha}=V^{\alpha \beta} X_{\beta}, Y_{\alpha}=Y^{\beta} V_{\beta \alpha}$.

$A d S_{5}$-полевая напряженность, соответствующая калибровочному полю (2.1), имеет вид

$$
R_{\alpha}^{\beta}=d \Omega_{\alpha}^{\beta}+\Omega_{\alpha}^{\gamma} \wedge \Omega_{\gamma}^{\beta}
$$

и фоновое пространство $A d S_{5}$ описывается полем 1-формы $\Omega_{0 \beta}^{\alpha}=\left(h^{\alpha \beta}, \omega_{0}^{\alpha(2)}\right)$, которая удовлетворяет условию нулевой кривизны [16]

$$
R_{\alpha}^{\beta}\left(\Omega_{0}\right)=0
$$

\section{3. ПОЛЯ ВЫСШИХ СПИнОВ}

Для того чтобы описать калибровочное поле спина $\left(s_{1}, s_{2}\right)$, введем пару взаимно сопряженных $\left.^{3}\right) s u(2,2)$-бесследовых мультиспиноров, симметричных по нижним и верхним индексам [1]-[6],

$$
\Omega_{\beta(n)}^{\alpha(m)}(x) \oplus \bar{\Omega}_{\alpha(m)}^{\beta(n)}(x), \quad \Omega^{\alpha(m-1) \gamma}{ }_{\beta(n-1) \rho}(x) \delta_{\gamma}^{\rho}=0,
$$

являющихся 1-формами

$$
\Omega_{\beta(n)}^{\alpha(m)}(x)=d x^{\underline{n}} \Omega_{\underline{n}}^{\alpha(m)}{ }_{\beta(n)}(x)
$$

$\mathrm{C}$

$$
m=s_{1}+s_{2}-1, \quad n=s_{1}-s_{2}-1 \text {. }
$$

Чтобы разложить представления (3.1) $A d S_{5}$-алгебры $s u(2,2)$ на представления ее лоренцевой подалгебры, мы используем компенсатор $V^{\alpha \beta}$. Результат редукции имеет вид

$$
\begin{aligned}
& \Omega^{\alpha\left(s_{1}+s_{2}-1\right)} \beta\left(s_{1}-s_{2}-1\right)(x)=\sum_{t=0}^{s_{1}-s_{2}-1} \omega^{\alpha\left(s_{1}+s_{2}-1\right) \gamma(t), \gamma\left(s_{1}-s_{2}-t-1\right)}(x) \times \\
& \times V_{\beta\left(s_{1}-s_{2}-1\right), \gamma\left(s_{1}-s_{2}-1\right)},
\end{aligned}
$$

3) Операция комплексного сопряжения определена правилом $\bar{X}_{\alpha}=X^{\beta} C_{\beta \alpha}, \bar{Y}^{\alpha}=C^{\alpha \beta} Y_{\beta}$, где черта означает комплексное сопряжение, а $C^{\alpha \beta}=-C^{\beta \alpha}$ и $C_{\alpha \beta}=-C_{\beta \alpha}$ есть некоторые вещественные матрицы, удовлетворяющие равенству $C_{\alpha \gamma} C^{\beta \gamma}=\delta_{\alpha}{ }^{\beta}$ [2]. 
где введено конденсированное обозначение $V_{\alpha(k), \beta(k)} \equiv V_{\alpha_{1} \beta_{1}} V_{\alpha_{2} \beta_{2}} \ldots V_{\alpha_{k} \beta_{k}}$. Неприводимые по отношению к алгебре Лоренца компоненты

$$
\omega^{\alpha\left(s_{1}+s_{2}+t-1\right), \beta\left(s_{1}-s_{2}-t-1\right)}(x), \quad 0 \leqslant t \leqslant s_{1}-s_{2}-1,
$$

удовлетворяют условию юнговой симметрии

$$
\omega^{\alpha\left(s_{1}+s_{2}+t-1\right), \alpha \beta\left(s_{1}-s_{2}-t-1\right)}(x)=0,
$$

и все свертки с $V_{\alpha \beta}$ являются нулями:

$$
\omega^{\alpha\left(s_{1}+s_{2}+t-1\right), \beta\left(s_{1}-s_{2}-t-1\right)}(x) V_{\alpha \beta}=0 .
$$

Согласно анализу, проведенному в работах [1], [2], мультиспиноры с $|m-n|=0$ соответствуют полностью симметричным бозонным полям спина $s_{1}$ и являются самосопряженными. Другие поля с $|m-n| \geqslant 1$ описываются парой взаимно сопряженных мультиспиноров и соответствуют либо полностью симметричным фермионным полям спина $s_{1},|m-n|=1$ [3], [4], либо бозонным и фермионным полям смешанного типа симметрии, $|m-n| \geqslant 2$ [2], [4]. Уместно сделать следующий комментарий: калибровочные поля смешанного типа симметрии с необходимостью появляются в спектре $\mathcal{N} \geqslant 2$ расширенных пятимерных калибровочных супералгебр высших спинов, в то время как $\mathcal{N} \leqslant 1$ (супер)алгебры описывают только полностью симметричные поля [11].

Для того чтобы связать спинорную и (спин-)тензорную формы динамики полей смешанного типа симметрии, рассмотрим $o(4,1)$-(спин-)тензорный вариант мультиспинорных полей $(3.5)-(3.6)$ при $s_{2} \neq 0$. Результат таков: набор $o(4,1)$-калибровочных полей представляется в виде комплекснозначных (спин-)тензорных полей вида

$$
\omega^{a\left(s_{1}-1\right), b\left(s_{2}+t\right)}=d x^{\underline{n}}{\omega_{\underline{n}}}^{a\left(s_{1}-1\right), b\left(s_{2}+t\right)}, \quad 0 \leqslant t \leqslant s_{1}-s_{2}-1,
$$

для бозонных полей смешанного типа симметрии и вида

$$
w^{\alpha \mid a\left(s_{1}-1\right), b\left(s_{2}+t\right)}=d x^{\underline{n}} w_{\underline{n}}^{\alpha \mid a\left(s_{1}-1\right), b\left(s_{2}+t\right)}, \quad \alpha=1,2,3,4, \quad 0 \leqslant t \leqslant s_{1}-s_{2}-1,
$$

для фермионных полей смешанного типа симметрии ( $\alpha$ обозначает пятимерный дираковский спинорный индекс). В обоих случаях поля (3.8), (3.9) удовлетворяют юнговскому свойству симметрии и являются бесследовыми (бозоны) или гаммапоперечными (фермионы).

Согласно терминологии работы [6], поля (3.8) и (3.9) с параметром $t \geqslant 1$ называются экстра полями. Фермионное поле (3.9) при $t=0$ будет называться физическим полем. Чтобы классифицировать бозонное поле (3.8) с $t=0$, мы разлагаем его на вещественную и мнимую части:

$$
\omega^{a\left(s_{1}-1\right), b\left(s_{2}\right)}=\omega_{1}^{a\left(s_{1}-1\right), b\left(s_{2}\right)}+i \omega_{2}^{a\left(s_{1}-1\right), b\left(s_{2}\right)} .
$$

С помощью пятимерного символа Леви-Чивита одно из полей $\left(\omega_{1}\right.$ или $\left.\omega_{2}\right)$ может быть дуализовано в поле с одним индексом в третьей строке, например,

$$
\omega^{a\left(s_{1}-1\right), b\left(s_{2}\right)}=\omega_{1}^{a\left(s_{1}-1\right), b\left(s_{2}\right)}+i \epsilon^{a b c d e} \omega_{2}^{a\left(s_{1}-2\right)} c,{ }^{b\left(s_{2}-1\right)} d, e,
$$


где дуальное поле с тремя строками $\omega_{2}^{a\left(s_{1}-1\right), b\left(s_{2}\right), e}$ является бесследовым и удовлетворяет юнговскому свойству симметрии. Мы называем вещественную часть поля (3.11) $\operatorname{Re} \omega^{a\left(s_{1}-1\right), b\left(s_{2}\right)}$ физическим полем, а его мнимую часть $\operatorname{Im} \omega^{a\left(s_{1}-1\right), b\left(s_{2}\right)}$ вспомогательным полем. На самом деле, любое бозонное поле (3.8) может быть представленно в виде пары вещественных полей, одно из которых имеет один индекс в третьей строке. Получающийся набор вещественных лоренц-ковариантных полей описывается трехстрочными $o(4,1)$-диаграммами Юнга, появляющимися как разложение определенной $o(4,2)$-трехстрочной диаграммы Юнга [2], [6].

\section{4. ЛИНЕАРИЗОВАННЫЕ КРИВИЗНЫ ВЫСШИХ СПИНОВ}

Анализ линеаризованых кривизн в этом разделе близок к аналогичному анализу, содержащемуся в предыдущих работах по полностью симметричным полям [2], [3]. Оказывается, что общий вид калибровочных преобразований для полей смешанного типа симметрии остается таким же, за исключением дополнительной зависимости от спина $s_{2}$ и появления ненулевого оператора $\mathcal{T}_{0}$ для бозонных несимметричных полей (см. (4.18), (4.20)). Это последнее свойство нетипично для бозонных систем и является отражением неявного присутствия символа Леви-Чивита в определении вещественных бозонных компонент комплекснозначных полей (3.8). Вычисление бозонного оператора $\mathcal{T}_{0}$ является основным результатом этого раздела.

Введем вспомогательные коммутирующие переменные $a_{\alpha}$ и $b^{\beta}$, преобразующиеся как фундаментальное и сопряженное фундаментальному представления $s u(2,2)$. Удобно представить поля высших спинов (3.2) в виде функций от вспомогательных переменных

$$
\Omega(a, b \mid x)=\Omega^{\alpha\left(s_{1}+s_{2}-1\right)}{ }_{\beta\left(s_{1}-s_{2}-1\right)}(x) a_{\alpha\left(s_{1}+s_{2}-1\right)} b^{\beta\left(s_{1}-s_{2}-1\right)},
$$

где

$$
a_{\alpha(m)}=a_{\alpha_{1}} \ldots a_{\alpha_{m}}, \quad b^{\beta(n)}=b^{\beta_{1}} \ldots b^{\beta_{n}} .
$$

Соответствующая пятимерная линеаризованная кривизна высших спинов имеет вид

$$
R(a, b \mid x)=d \Omega(a, b \mid x)+\Omega_{0}{ }^{\alpha}{ }_{\beta}\left(b^{\beta} \frac{\partial}{\partial b^{\alpha}}-a_{\alpha} \frac{\partial}{\partial a_{\beta}}\right) \wedge \Omega(a, b \mid x),
$$

где $\Omega_{0}{ }^{\alpha}{ }_{\beta}$ - фоновая 1-форма связности, удовлетворяющая условию нулевой кривизны (2.4). Линеаризованные (абелевы) преобразования высших спинов есть

$$
\delta \Omega(a, b \mid x)=D_{0} \xi(a, b \mid x)
$$

где фоновая ковариантная производная имеет вид

$$
D_{0}=d+\Omega_{0}{ }^{\alpha}{ }_{\beta}\left(b^{\beta} \frac{\partial}{\partial b^{\alpha}}-a_{\alpha} \frac{\partial}{\partial a_{\beta}}\right) .
$$

Из условия (2.4) следует, что $\delta R(a, b \mid x)=0$. Тождества Бианки имеют вид

$$
D_{0} R(a, b \mid x)=0 \text {. }
$$


В последующем анализе мы используем два набора дифференциальных операторов по вспомогательным переменным [2] -

$$
S^{-}=a_{\alpha} \frac{\partial}{\partial b^{\beta}} V^{\alpha \beta}, \quad S^{+}=b^{\alpha} \frac{\partial}{\partial a_{\beta}} V_{\alpha \beta}, \quad S^{0}=N_{b}-N_{a}
$$

и

$$
T^{-}=\frac{1}{4} \frac{\partial^{2}}{\partial a_{\alpha} \partial b^{\alpha}}, \quad T^{+}=a_{\alpha} b^{\alpha}, \quad T^{0}=\frac{1}{4}\left(N_{a}+N_{b}+4\right),
$$

где

$$
N_{a}=a_{\alpha} \frac{\partial}{\partial a_{\alpha}}, \quad N_{b}=b^{\alpha} \frac{\partial}{\partial b^{\alpha}} .
$$

$\mathrm{C}$ помощью (4.7) и (4.8) условия неприводимости для $\Omega(a, b)$ переформулируются в виде

$$
T^{-} \Omega(a, b)=0, \quad\left(S^{0}+2 s_{2}\right) \Omega(a, b)=0 .
$$

Как показано в разделе 3 , калибровочное поле высшего спина $\Omega$ разлагается на представления лоренцевой подалгебры согласно формуле (3.4). В терминах операторов (4.7) и (4.8) формула (3.4) переписывается в виде

$$
\Omega(a, b \mid x)=\sum_{t=0}^{s_{1}+s_{2}-1}\left(S^{+}\right)^{t} \omega^{t}(a, b \mid x),
$$

где

$$
\omega^{t}(a, b \mid x)=\omega^{\alpha\left(s_{1}+s_{2}+t-1\right), \beta\left(s_{1}-s_{2}-t-1\right)}(x) a_{\alpha\left(s_{1}+s_{2}+t-1\right)} b_{\beta\left(s_{1}-s_{2}-t-1\right)}
$$

суть лоренц-ковариантные калибровочные поля (3.5), и условия неприводимости (3.6), (3.7) принимают вид

$$
S^{-} \omega^{t}(a, b)=0, \quad T^{-} \omega^{t}(a, b)=0 .
$$

Калибровочная симметрия высших спинов (4.4) требует, чтобы как бозонные, так и фермионные лоренц-ковариантные кривизны высших спинов $r^{t}$ и калибровочные преобразования имели следующий вид:

$$
\begin{aligned}
r^{t} & =\mathcal{D} \omega^{t}+\mathcal{T}^{-} \omega^{t+1}+\lambda \mathcal{T}^{0} \omega^{t}+\lambda^{2} \mathcal{T}^{+} \omega^{t-1}, \\
\delta \omega^{t} & =\mathcal{D} \xi^{t}+\mathcal{T}^{-} \xi^{t+1}+\lambda \mathcal{T}^{0} \xi^{t}+\lambda^{2} \mathcal{T}^{+} \xi^{t-1},
\end{aligned}
$$

где 0-формы $\xi^{t}$ - лоренц-ковариантные калибровочные параметры и $\mathcal{D}$ - фоновая лоренц-ковариантная производная

$$
\mathcal{D}=d+w_{0 \beta}^{\alpha}\left(a_{\alpha} \frac{\partial}{\partial a_{\beta}}+b_{\alpha} \frac{\partial}{\partial b_{\beta}}\right) .
$$

Операторы $\mathcal{T}^{-}, \mathcal{T}^{+}$и $\mathcal{T}^{0}$ имеют вид

$$
\mathcal{T}^{+}=\left(1-\frac{\Delta^{2}}{\left(S^{0}\right)^{2}}\right) h^{\alpha}{ }_{\beta} a_{\alpha} \frac{\partial}{\partial b_{\beta}},
$$




$$
\begin{aligned}
\mathcal{T}^{0}=- & \frac{\Delta}{S^{0}} h^{\alpha}{ }_{\beta}\left(b_{\alpha} \frac{\partial}{\partial b_{\beta}}-a_{\alpha} \frac{\partial}{\partial a_{\beta}}+\frac{2}{S^{0}-2}\left(b_{\gamma} \frac{\partial}{\partial a_{\gamma}}\right) a_{\alpha} \frac{\partial}{\partial b_{\beta}}\right), \\
\mathcal{T}^{-}= & \frac{1}{1-S^{0}} h^{\alpha}{ }_{\beta}\left(\left(2-S^{0}\right) b_{\alpha} \frac{\partial}{\partial a_{\beta}}+b_{\gamma} \frac{\partial}{\partial a_{\gamma}}\left(b_{\alpha} \frac{\partial}{\partial b_{\beta}}-a_{\alpha} \frac{\partial}{\partial a_{\beta}}\right)+\right. \\
& \left.+\frac{1}{S^{0}-3}\left(b_{\gamma} \frac{\partial}{\partial a_{\gamma}}\right)^{2} a_{\alpha} \frac{\partial}{\partial b_{\beta}}\right),
\end{aligned}
$$

где параметр $\Delta$ принимает значения

$$
\Delta= \begin{cases}2 s_{2} & \text { для бозонов, } \\ 2 s_{2}+1 & \text { для фермионов }\end{cases}
$$

и удовлетворяют следующим соотношениям:

$$
\begin{gathered}
\left\{\mathcal{T}^{0}, \mathcal{T}^{-}\right\}=\left\{\mathcal{T}^{0}, \mathcal{T}^{+}\right\}=0, \\
\left(\mathcal{T}^{-}\right)^{2}=0, \quad\left(\mathcal{T}^{+}\right)^{2}=0, \\
\mathcal{D}^{2}+\lambda^{2}\left\{\mathcal{T}^{-}, \mathcal{T}^{+}\right\}+\lambda^{2}\left(\mathcal{T}^{0}\right)^{2}=0 .
\end{gathered}
$$

Отметим, что коэффициенты в (4.17)-(4.19) могут быть изменены переопределением полей вида $\widetilde{\omega}^{t}=C(t, s) \omega^{t}$ при $C \neq 0$.

\section{5. ДЕЙСТВИЕ ВЫСШИХ СПИНОВ}

Прежде чем рассматривать действия для произвольных калибровочных полей смешанного типа симметрии, посмотрим, что происходит в случае простейшего несимметричного бозонного поля спина $(2,1)$, описываемого 1-формой $\Omega^{\alpha(2)}(x)$. С точностью до вкладов с полными производными функционал действия имеет единственный вид

$$
\mathcal{S}_{2}^{(2,1)}=\int_{\mathcal{M}^{5}} h_{\beta}^{\alpha} \wedge R^{\beta \gamma} \wedge \bar{R}_{\gamma \alpha},
$$

где $h_{\beta}^{\alpha}$ есть фоновое $A d S_{5}$-поле репера и кривизна задается в виде

$$
R^{\alpha(2)}=D_{0} \Omega^{\alpha(2)} \equiv \mathcal{D} \Omega^{\alpha(2)}+\lambda h_{\gamma}^{\alpha} \wedge \Omega^{\gamma \alpha}
$$

Уравнения движения, получающиеся при варьировании действия (5.1), таковы:

$$
H_{2}{ }^{\alpha} \wedge R^{\gamma \beta}+H_{2}{ }^{\beta}{ }_{\gamma} \wedge R^{\gamma \alpha}=0,
$$

плюс комплексно-сопряженные уравнения для $\bar{\Omega}_{\alpha \beta}$. Отметим, что эти бозонные уравнения являются уравнениями 1-го порядка, что делает их похожими на фермионные уравнения. Однако, как обсуждалось в разделе 3 , вещественная и мнимая части комплекснозначного поля $\Omega^{\alpha(2)}(x)$ рассматриваются как физическое и вспомогательное поля (3.11), и вспомогательное поле выражается посредством своего уравнения движения через первые производные физического поля. Для того чтобы дать более детальное описание этого механизма, рассмотрим тензорную форму 
действия (5.1). Согласно (3.10) o(4,1)-поле, изоморфное полю $\Omega^{\alpha(2)}(x)$, задается формулой

$$
\omega^{[a b]}=\omega_{1}^{a b}+i \omega_{2}^{a b} .
$$

Соответствующая линеаризованная кривизна и калибровочные преобразования имеют вид

$$
R^{a b}=\mathcal{D} \omega^{a b}-\frac{i \lambda}{2} \epsilon^{a b c d e} h_{c} \wedge \omega_{d e}, \quad \delta \omega^{a b}=\mathcal{D} \xi^{a b}-\frac{i \lambda}{2} \epsilon^{a b c d e} h_{c} \xi_{d e},
$$

где $\mathcal{D}$ - фоновая лоренц-ковариантная производная, $\xi^{a b}-0$-форма комплексного калибровочного параметра и $h^{a}$ - фоновое поле репера. Отметим, что слагаемые в (5.5), содержащие символ Леви-Чивита, фактически являются оператором $\mathcal{T}^{0}$, заданным в спинорных обозначениях формулой (4.18). Тождества Бианки имеют вид

$$
\mathcal{D} R^{a b}-\frac{i \lambda}{2} \epsilon^{a b c d e} h_{c} \wedge R_{d e}=0 .
$$

Действие можно записать аналогично выражению (5.5) как

$$
\mathcal{S}_{2}^{(2,1)}=\int_{\mathcal{M}^{5}} \epsilon_{a b c d e} h^{e} \wedge R^{a b} \wedge \bar{R}^{c d}
$$

где $\bar{R}^{c d}$ - комплексно-сопряженная кривизна (5.5). Уравнения движения суть

$$
H_{a}{ }^{c} \wedge R_{c b}-H_{b}{ }^{c} \wedge R_{c a}=0, \quad H_{a b} \stackrel{\text { def }}{=} h_{a} \wedge h_{b}
$$

плюс комплексно-сопряженные уравнения. Чтобы выявить динамическое содержание этих уравнений, вещественная либо мнимая часть поля $\omega^{a b}(5.4)$ рассматривается как дуализованное вспомогательное поле, например,

$$
\omega_{1}^{a b}=\omega_{1}^{a b}, \quad \omega_{2}^{a b}=\frac{1}{\lambda} \epsilon^{a b c d e} \omega_{2} c d e,
$$

где $\omega_{1}^{a b}$ и $\omega_{2}^{a b c}$ - физическое и вспомогательное поля с антисимметричными индексами и множитель $\lambda^{-1}$ введен для выражения того факта, что массовые размерности физического и вспомогательного полей различны. Эти поля могут быть объединены в одно $o(4,2)$-поле $\Omega^{[A B C]}[6]$. Можно показать, что действие (5.7) может быть переписано как

$$
\mathcal{S}_{2}^{(2,1)}=\frac{1}{\lambda^{2}} \int_{\mathcal{M}^{d}} \epsilon_{A B C D E F} h^{E} V^{F} \wedge R^{A B M} \wedge R^{C D N} V_{M} V_{N},
$$

и в этом виде оно совпадает с действием для $A d S_{d}$-поля "крюка", подробно изученным в работе [6]. Стоит отметить, что плоский предел действия (5.7) (или, эквивалентно, действия (5.10)) приводит к дуальному описанию поля спина 2, что в точности соответствует действию Картрайта [17]. Заметим также, что процедура объединения физического и вспомогательного полей в одно комплекснозначное поле была использована при изучении так называемой самодуальности в нечетных размерностях для массивных антисимметричных тензорных полей в пространстве Минковского [18]. 
Действие для несимметричных $A d S_{5}$ калибровочных полей. Далее мы строим свободные действия, описывающие бозонные и фермионные калибровочные поля смешанного типа симметрии в $A d S_{5}$. Случай полностью симметричных полей был рассмотрен в работах [2], [3].

Так же, как в работах [2], [3], [6], мы ищем функционалы действия для полей смешанного типа симметрии в виде

$$
\mathcal{S}_{2}^{\left(s_{1}, s_{2}\right)}=\left.\int_{\mathcal{M}^{5}} \widehat{H} \wedge R^{s_{1}, s_{2}}\left(a_{1}, b_{1}\right) \wedge \bar{R}^{s_{1}, s_{2}}\left(a_{2}, b_{2}\right)\right|_{a_{i}=b_{i}=0},
$$

где $R^{s_{1}, s_{2}}$ - линеаризованная кривизна высшего спина (4.3) и 1-форма $\widehat{H}$ является следующим дифференциальным оператором:

$$
\begin{aligned}
\widehat{H}=( & \alpha(p, q) h_{\alpha \beta} \frac{\partial^{2}}{\partial a_{1 \alpha} \partial a_{2 \beta}} \hat{b}_{12}+\beta(p, q) h^{\alpha \beta} \frac{\partial^{2}}{\partial b_{1}^{\alpha} \partial b_{2}^{\beta}} \hat{a}_{12}+ \\
& \left.+\gamma(p, q) h_{\alpha}{ }^{\beta} \frac{\partial^{2}}{\partial a_{2 \alpha} \partial b_{1}^{\beta}} \hat{c}_{12}+\zeta(p, q) h_{\alpha}{ }^{\beta} \frac{\partial^{2}}{\partial a_{1 \alpha} \partial b_{2}^{\beta}} \hat{c}_{21}\right)\left(\hat{c}_{12}\right)^{2 s_{2}} .
\end{aligned}
$$

Здесь $h_{\alpha}{ }^{\beta}$ - фоновое поле репера и коэффициенты $\alpha, \beta, \gamma$ и $\zeta$ являются функциями операторов

$$
p=\hat{a}_{12} \hat{b}_{12}, \quad q=\hat{c}_{12} \hat{c}_{21},
$$

где

$$
\begin{array}{ll}
\hat{a}_{12}=V_{\alpha \beta} \frac{\partial^{2}}{\partial a_{1 \alpha} \partial a_{2 \beta}}, & \hat{b}_{12}=V^{\alpha \beta} \frac{\partial^{2}}{\partial b_{1}^{\alpha} \partial b_{2}^{\beta}}, \\
\hat{c}_{12}=\frac{\partial^{2}}{\partial a_{1 \alpha} \partial b_{2}^{\alpha}}, & \hat{c}_{21}=\frac{\partial^{2}}{\partial a_{2 \alpha} \partial b_{1}^{\alpha}} .
\end{array}
$$

Эти функции отвечают за различные типы индексных сверток между полем репера и кривизнами. Действие инвариантно относительно комплексного сопряжения $\overline{\mathcal{S}}_{2}=$ $\mathcal{S}_{2}$ при вещественных коэффициентах $\alpha, \beta, \gamma$ и $\zeta$.

Так как вариация общего вида линеаризованных кривизн имеет вид $\delta R=D_{0} \delta \Omega$ и действие сформулировано $A d S_{5}$-ковариантным образом, при интегрировании по частям получается вариация

$$
\delta \mathcal{S}_{2}^{\left(s_{1}, s_{2}\right)}=\left.\int_{\mathcal{M}^{5}} D_{0} \widehat{H} \wedge \delta \Omega\left(a_{1}, b_{1}\right) \wedge \bar{R}\left(a_{2}, b_{2}\right)\right|_{a_{i}=b_{i}=0}+\text { K.c. }
$$

Производная $D_{0}$ дает поле репера каждый раз при действии на компенсатор, $D_{0} V^{\alpha \beta}$ $=h^{\alpha \beta}$. Учитывая $D_{0} h^{\alpha \beta}=0, h_{\alpha}{ }^{\beta}=h_{\alpha \gamma} V^{\beta \gamma}$ и обозначая $H^{\alpha \beta}=H^{\beta \alpha}=h_{\gamma}^{\alpha} \wedge h^{\beta \gamma}$, находим

$$
\begin{aligned}
D_{0} \widehat{H}= & \left(\rho_{1} H_{\alpha}{ }^{\beta} \frac{\partial^{2}}{\partial a_{2 \alpha} \partial b_{1} \beta} \hat{c}_{12}+\rho_{2} H_{\alpha}{ }^{\beta} \frac{\partial^{2}}{\partial a_{1 \alpha} \partial b_{2}{ }^{\beta}} \hat{c}_{21}+\right. \\
& \left.+\rho_{3} H_{\alpha \beta} \frac{\partial^{2}}{\partial a_{1 \alpha} \partial a_{2 \beta}} \hat{b}_{12}+\rho_{3} H^{\alpha \beta} \frac{\partial^{2}}{\partial b_{1}^{\alpha} \partial b_{2}^{\beta}} \hat{a}_{12}\right)\left(\hat{c}_{12}\right)^{2 s_{2}},
\end{aligned}
$$


где

$$
\begin{aligned}
& \rho_{1}=\frac{1}{2}\left(1+p \frac{\partial}{\partial p}\right)(-2 \gamma(p, q)+(\alpha+\beta)(p, q)), \\
& \rho_{2}=\frac{1}{2}\left(1+p \frac{\partial}{\partial p}\right)(-2 \zeta(p, q)+(\alpha+\beta)(p, q)), \\
& \rho_{3}=\frac{1}{2} q \frac{\partial}{\partial p}(\zeta(p, q)-\gamma(p, q)) .
\end{aligned}
$$

Для тривиального решения $\rho_{i}=0$, ковариантная производная от $\widehat{H}$ равна нулю, $D_{0} \widehat{H}=0$, и соответствующий функционал действия является полной производной. Из (5.17) следует, что $\rho_{i}=0$ при условии

$$
(\alpha+\beta)(p, q)=2 \gamma(p, q), \quad \zeta(p, q)=\gamma(p, q) .
$$

Очевидно, что добавлением полных производных с коэффициентами, удовлетворяющими (5.18), мы всегда можем положить $\gamma=0$ и $\beta=0$ в действии (5.11), (5.12).

В целом действие (5.11) не описывает безмассовые поля высших спинов по причине слишком большого количества нефизических динамических переменных, ассоциированных с экстра полями. Для того чтобы исключить соответствующие степени свободы, следует зафиксировать оператор $\widehat{H}$ подходящим образом, наложив условие отщепления [6], [19]. Это условие требует, чтобы вариация квадратичного действия по отношению к экстра полям была тождественно равна нулю:

$$
\frac{\delta \mathcal{S}_{2}^{\left(s_{1}, s_{2}\right)}}{\delta \omega^{t>0}} \equiv 0 .
$$

Чтобы проанализировать условие отщепления экстра полей, отметим, что все калибровочные поля экстра типа могут быть объединены в один неприводимый $s u(2,2)$ тензор $\xi(a, b)$, удовлетворяющий уравнению $\left(N_{a}-N_{b}-2 s_{2}-2\right) \xi(a, b)=0$. Тогда вариация экстра полей принимает вид

$$
\delta \Omega^{\text {extra }}(a, b)=S^{+} \xi(a, b),
$$

и условие отщепления экстра полей (5.19) сводится к

$$
\begin{gathered}
\left(\frac{\partial}{\partial p}-\frac{\partial}{\partial q}\right)\left(q \rho_{2}\right)+\rho_{3}=0 \\
\left(\frac{\partial}{\partial p}-\frac{\partial}{\partial q}\right) \rho_{3}=0 \\
\rho_{1}+\rho_{3}=0 .
\end{gathered}
$$

По модулю вкладов с полными производными (5.18) общее решение системы (5.21) имеет вид

$$
\begin{aligned}
\gamma(p, q) & =0, \quad \beta(p, q)=0, \quad \zeta(p, q)=\zeta^{(0)} \frac{(p+q)^{s_{1}-s_{2}-1}}{q} \\
\alpha(p, q) & =-\zeta^{(0)}\left(s_{1}-s_{2}-1\right) \int_{0}^{1} d \tau(p \tau+q)^{s_{1}-s_{2}-2}= \\
& =-\zeta^{(0)} \sum_{k=0}^{s_{1}-s_{2}-2} \frac{\left(s_{1}-s_{2}-1\right) !}{(k+1) !\left(s_{1}-s_{2}-k-2\right) !} p^{k} q^{s_{1}-s_{2}-k-2}
\end{aligned}
$$


Появление $q^{-1}$ в $\zeta(p, q)$ может быть удалено переопределением $\zeta(p, q) \rightarrow q \zeta(p, q)$. Эта операция действительно удаляет сингулярность, потому что последнее слагаемое в операторе $\widehat{H}(5.12)$ содержит комбинацию $\hat{c}_{21}\left(\hat{c}_{12}\right)^{2 s_{2}}$, которая по определению $q(5.14)$ всегда имеет вид $q\left(\hat{c}_{12}\right)^{2 s_{2}-1}$. Общий фактор $\zeta^{(0)}$ перед действием данного спина $\left(s_{1}, s_{2}\right)$ не может быть зафиксирован при анализе свободного действия и является остаточным произволом в коэффициентах.

\section{6. УРАВНЕНИЯ ДВИЖЕНИЯ И СВЯЗИ}

Для получения уравнений движения нетривиальная часть вариации (5.15) переписывается в следующем виде:

$$
\begin{aligned}
\delta \mathcal{S}_{2}^{\left(s_{1}, s_{2}\right)}=- & \frac{\zeta^{(0)}\left(s_{1}-s_{2}-1\right)}{2} \int(p+q)^{s_{1}-s_{2}-2}\left(\frac{\left(s_{1}-s_{2}+1\right) q+2\left(s_{1}-s_{2}\right) p}{s_{1}-s_{2}-1} \times\right. \\
& \times H_{\alpha}{ }^{\beta} \frac{\partial^{2}}{\partial a_{1 \alpha} \partial b_{2}{ }^{\beta}}+H_{\alpha}{ }^{\beta} \frac{\partial^{2}}{\partial a_{2 \alpha} \partial b_{1} \beta}\left(\hat{c}_{12}\right)^{2}-H_{\alpha \beta} \frac{\partial^{2}}{\partial a_{1 \alpha} \partial a_{2 \beta}} \hat{b}_{12} \hat{c}_{12}- \\
& \left.-H^{\alpha \beta} \frac{\partial^{2}}{\partial b_{1}^{\alpha} \partial b_{2}^{\beta}} \hat{a}_{12} \hat{c}_{12}\right)\left(\hat{c}_{12}\right)^{2 s_{2}-1} \wedge r^{0}\left(a_{1}, b_{1}\right) \wedge \delta \bar{\omega}^{0}\left(a_{2}, b_{2}\right)+\text { k.c. }
\end{aligned}
$$

Подставляя в вариацию (6.1) поля

$$
\begin{aligned}
r^{0}\left(a_{1}, b_{1}\right) & =r^{0 \alpha\left(s_{1}+s_{2}-1\right),}{ }_{\beta\left(s_{1}-s_{2}-1\right)} a_{1 \alpha\left(s_{1}+s_{2}-1\right)} b_{1}^{\beta\left(s_{1}-s_{2}-1\right)}, \\
\bar{\omega}^{0}\left(a_{2}, b_{2}\right) & =\bar{\omega}_{\gamma\left(s_{1}+s_{2}-1\right)}{ }^{\rho\left(s_{1}-s_{2}-1\right)} a_{2 \rho\left(s_{1}-s_{2}-1\right)} b_{2}^{\gamma\left(s_{1}+s_{2}-1\right)}
\end{aligned}
$$

и пользуясь их свойствами юнговской симметрии

$$
\begin{aligned}
Y_{1} \equiv S_{1}^{-}: & Y_{1} r^{0}\left(a_{1}, b_{1}\right)=0, \\
Y_{2} \equiv S_{2}^{+}: & Y_{2} \bar{\omega}^{0}\left(a_{2}, b_{2}\right)=0,
\end{aligned}
$$

получаем уравнения движения, которые могут быть представлены в следующем удобном виде:

$$
\widehat{E} \wedge r^{0}(a, b)=0
$$

где $\widehat{E}-2$-форма, являющаяся дифференциальным оператором вида

$$
\widehat{E}=H_{\beta}^{\alpha}\left(a_{\alpha} \frac{\partial}{\partial a_{\beta}}+\kappa_{2} b_{\alpha} \frac{\partial}{\partial b_{\beta}}+\kappa_{3} S^{+} a_{\alpha} \frac{\partial}{\partial b_{\beta}}+\kappa_{4} T^{+} \frac{\partial^{2}}{\partial a^{\alpha} \partial b_{\beta}}+\kappa_{5} T^{+} S^{+} \frac{\partial^{2}}{\partial b^{\alpha} \partial b_{\beta}}\right)
$$

с коэффициентами

$$
\kappa_{2}=\frac{1+\left(s_{1}+s_{2}-1\right)\left(s_{2}+1\right)}{1-\left(s_{1}-s_{2}+1\right)\left(s_{2}+1\right)}, \quad \kappa_{3}=-\kappa_{4}=\frac{1-\kappa_{2}}{2\left(s_{2}+1\right)}, \quad \kappa_{5}=\frac{\kappa_{2}-1}{4 s_{1}\left(s_{2}+1\right)} .
$$

Аналогичные уравнения имеют место для комплексно-сопряженного физического поля $\bar{\omega}^{0}$. Оператор $\widehat{E}$ удовлетворяет условиям

$$
\left[S^{-}, \widehat{E}\right]=0, \quad\left[T^{-}, \widehat{E}\right]=0,
$$


т.е. сохраняет свойства юнговской симметрии и $V$-поперечности физической кривизны $r^{0}$. Также по построению он удовлетворяет условию отщепления экстра полей, означающему, что слагаемое $\mathcal{T}^{-} \omega^{1}$, содержащее экстра поле $\omega^{1}$ в кривизне $r^{0}=\mathcal{D} \omega^{0}+\mathcal{T}^{0} \omega^{0}+\mathcal{T}^{-} \omega^{1}$, не дает вклада в уравнения движения, т.е. $\widehat{E} \wedge \mathcal{T}^{-} \omega^{1}=0$.

Так же, как в работах по полностью симметричным полям [19], мы полагаем, что связи для экстра полей должны иметь вид

$$
\Upsilon_{2}^{+} \wedge r^{t}(a, b)=0, \quad 0 \leqslant t<s_{1}-s_{2}-1,
$$

где 2-форма $\Upsilon_{2}^{+}$является оператором, который увеличивает $t$ и удовлетворяет условию

$$
\mathcal{T}^{+} \wedge \Upsilon_{2}^{+}=0
$$

Требуется, чтобы оператор $\Upsilon_{2}^{+}$обладал свойством (6.9), так как это гарантирует совпадение числа независимых алгебраических соотношений для кривизны $r^{t}$ с числом компонент экстра полей $\omega^{t>0}$ по модулю чисто калибровочных компонент вида $\delta \omega^{t+1}=\mathcal{T}^{-} \epsilon^{t+2}$. Можно показать, что оператор $\Upsilon_{2}^{+}$единственным образом фиксируется в виде

$$
\Upsilon_{2}^{+}=\mathcal{T}^{0} \wedge \mathcal{T}^{+}
$$

На основе связей (6.8) поле $\omega^{t+1}$ может быть выражено через производные поля $\omega^{t}$ для любого $t>0$. И, наконец, можно получить поля $\omega^{t}$, выраженные в терминах производных поля $\omega^{0}$ с порядком старшей производной, равным $t$.

\section{7. ЗАКЛЮЧЕНИЕ}

В этой статье мы построили явно ковариантную лагранжеву формулировку для $A d S_{5}$ безмассовых калибровочных полей смешанного типа симметрии в рамках $s u(2,2)$-спинорного формализма. Подход, который мы использовали, основан на реперной формулировке полей смешанного типа симметрии, разработанной в работах [6], [7]. Наши результаты могут рассматриваться как окончательный шаг в изучении явно ковариантной лагранжевой формулировки $A d S_{5}$ калибровочных полей высших спинов в $s u(2,2)$-формализме. Важной проблемой для дальнейших исследований является развитие развернутой формы динамики свободных полей смешанного типа симметрии, основанной на тензорах Вейля, возникающих из уравнений движения и связей для экстра полей, проанализированных в разделе 6 . Это позволит сформулировать центральную теорему массовой поверхности подобно случаю полностью симметричных калибровочных полей [2], [19] и установить связь с развернутой формулировкой полей смешанного типа симметрии, развитой в работе [4]. Построенная лагранжева формулировка делает также возможным изучение $\mathcal{N}$-расширенных суперсимметричных кубических взаимодействий $A d S_{5}$ калибровочных полей на уровне функционалов действия, обобщая, таким образом, $\mathcal{N}=0,1$ результаты работ [2], [5].

Благодарности. Автор признателен М. А. Васильеву за полезные обсуждения. Работа частично поддержана РФФИ (грант № 02-02-16944), Программой поддержки ведущих научных школ (грант № НШ-1578-2003-2), INTAS (грант № 03-51-6346). 


\section{Список литературы}

[1] E. Sezgin, P. Sundell, JHEP, 2001, № 09, 036; hep-th/0105001.

[2] M. A. Vasiliev, Nucl. Phys. B, 616 (2001), 106; Erratum, 652 (2003), 407; hep-th/0106200.

[3] K. B. Alkalaev, Phys. Lett. B, 519 (2001), 121; hep-th/0107040.

[4] E. Sezgin, P. Sundell, JHEP, 2001, № 09, 025; hep-th/0107186.

[5] K. B. Alkalaev, M. A. Vasiliev, Nucl. Phys. B, 655 (2003), 57; hep-th/0206068.

[6] K. B. Alkalaev, O. V. Shaynkman, M. A. Vasiliev, Nucl. Phys. B, 692 (2004), 363; hep-th/0311164; К. Б. Алкалаев, ТMФ, 140 (2004), 424; hep-th/0311212.

[7] K. B. Alkalaev, O. V. Shaynkman, M. A. Vasiliev, JHEP, 2005, № 08, 069; hep-th/0501108.

[8] E. Sezgin, P. Sundell, Nucl. Phys. B, 644 (2002), 303; Err., 660 (2003), 403; hep-th/0205131; M. Bianchi, Fortschr. Phys., 53 (2005), 665; hep-th/0409304.

[9] M. A. Vasiliev, Phys. Lett. B, 567 (2003), 139; hep-th/0304049; JHEP, 2004, № 12, 046; hep-th/0404124.

[10] E. S. Fradkin, V. Y. Linetsky, Mod. Phys. Lett. A, 4 (1989), 2363.

[11] M. A. Vasiliev, Phys. Rev. D, 66 (2002), 066006; hep-th/0106149.

[12] R. R. Metsaev, Phys. Lett. B, 354 (1995), 78; 419 (1998), 49; hep-th/9802097; Arbitrary spin massless bosonic fields in d-dimensional anti-de Sitter space, hep-th/9810231.

[13] R. R. Metsaev, Phys. Lett. B, 531 (2002), 152; hep-th/0201226.

[14] L. Brink, R. R. Metsaev, M. A. Vasiliev, Nucl. Phys. B, 586 (2000), 183; hep-th/0005136; T. Biswas, W. Siegel, JHEP, 2002, № 07, 005; hep-th/0203115; Yu. M. Zinoviev, On massive mixed symmetry tensor fields in Minkowski space and $(A) d S$, hep-th/0211233; First order formalism for mixed symmetry tensor fields, hep-th/0304067; P. de Medeiros, Class. Quant. Grav., 21 (2004), 2571; hep-th/0311254.

[15] K. Stelle, P. West, Phys. Rev. D, 21 (1980), 1466; C. R. Preitschopf, M. A. Vasiliev, "The superalgebraic approach to supergravity", Proc. 31th Intern. Ahrenshoop Symp. on the Theory of Elementary Particles (Buckow, September 2-6, 1997), eds. H. Dorn, D. Lüst, G. Weight, Wiley-VCH, Weinheim, 1998, 483; hep-th/9805127.

[16] M. A. Vasiliev, "Higher spin gauge theories: star-product and AdS Space", "The Many Faces of the Superworld", Yuri Golfand Memorial Volume, ed. M. Shifman, World Scientific, Singapore, 2000, 533; hep-th/9910096.

[17] T. Curtright, Phys. Lett. B, 165 (1985), 304.

[18] P. K. Townsend, K. Pilch, P. van Nieuwenhuizen, Phys. Lett. B, 136 (1984), 38; Add., 137 (1984), 443.

[19] V.E. Lopatin, M. A. Vasiliev, Mod. Phys. Lett. A, 3 (1988), 257; M. A. Vasiliev, Nucl. Phys. B, 301 (1988), 26. 